\title{
Cortical Activation during Walking While Smartphone Texting: a Dual Task \\ Based fNRIS Study
}

Kun Wang, Zhangyan Deng, Jimeng Zhang, Qian Gu, Tao Huang, Zuosong Chen Department of Physical Education, Shanghai Jiao Tong University, Shanghai , China

\section{Objectives}

The purpose of the study was to investigate the cortical activation during a dual task of walking while smartphone texting in young healthy adults using the functional nearinfrared spectroscopy (fNIRS).

\section{Methods}

In a crossover study design, 39 righthanded college students $(21.3 \pm 2.5$ years, $46.1 \%$ females) randomly undertook the following three task conditions separated by a minimum of 48 hours: smartphone texting only ( $T$ task), walking only (W task), and dual task of smartphone texting while walking (TW task). Cortical oxygenation during the three tasks was monitored using a 38-channel fNIRS (NIRx Medical Technologies LLC, USA). Walking was conducted on a treadmill with a speed of $2.0 \mathrm{~km} /$ hour. Texting task was performed with a typing APP on a smartphone.

\section{Results}

There was no significant difference in texting speed between $T$ task and TW task (71.7 \pm 10.4 vs. 71.0 \pm 12.9 chars $/ \mathrm{min}, \mathrm{P}>0.05$ ).

Texting enhanced hemodynamic response in frontopolar area (e.g., Ch1T task vs. W task, $1.10 \pm 1.93$ vs. $-0.12 \pm 0.14$ a.u, $P<0.01$; Ch1TW task vs. W task $0.89 \pm 1.05$ vs. $-0.12 \pm 0.14$ a.u, $P<$ 0.01) dorsolateral prefrontal cortex (e.g., Ch6T task vs. $W$ task, $0.24 \pm 0.43$ vs. $-0.15 \pm 0.42$ a.u, $P<$ 0.01 ; Ch6TW task vs. W task $0.21 \pm 0.33$ vs. $0.15 \pm 0.42$ a.u, $P<0.01$ ) and Broca's area (e.g., Ch35TW task vs. W task $0.99 \pm 0.81$ vs. $0.47 \pm 0.75$ a.u, $\mathrm{P}<0.01$ ).

\section{Texting vs. Walking}

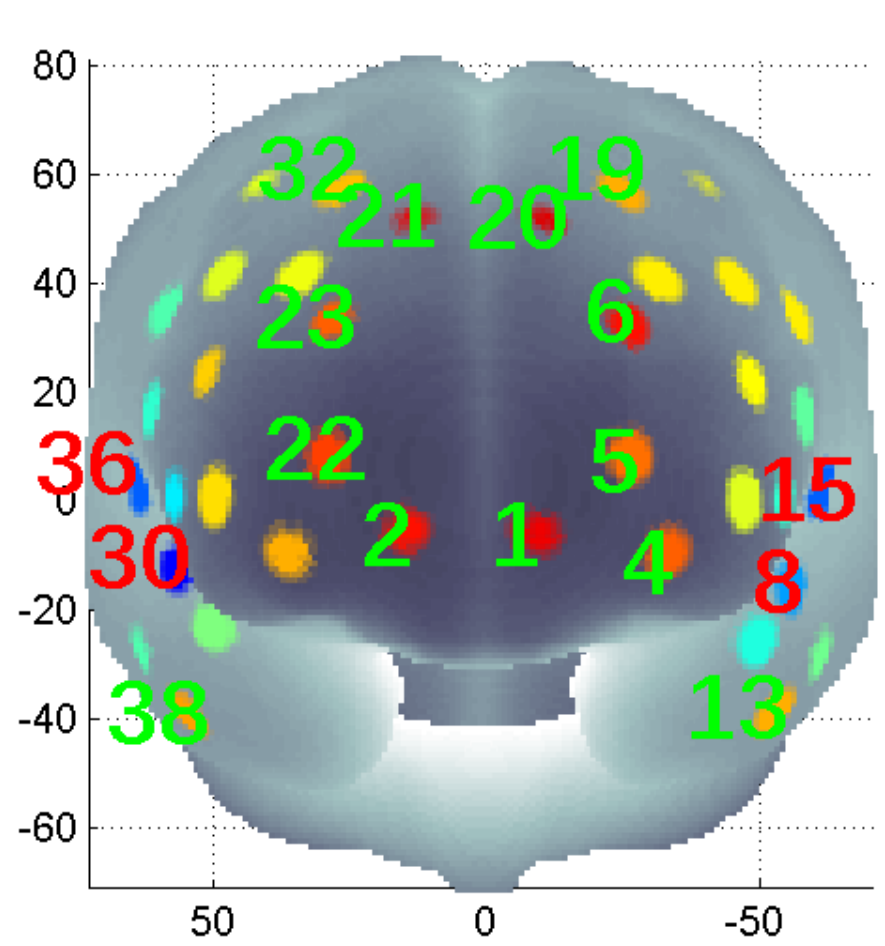

T-value

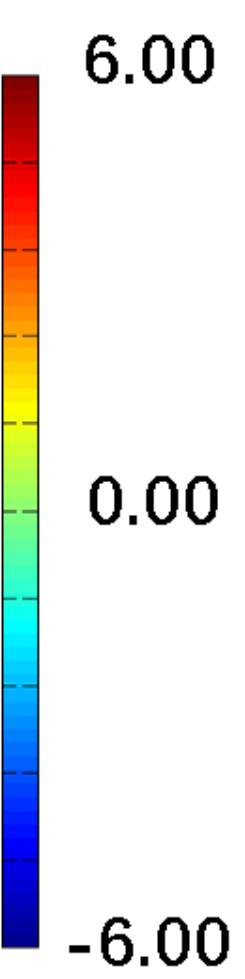

In addition, $\mathrm{W}$ task evoked an increased activation in temporopolar area (e.g., Ch8 T task vs. W task $0.04 \pm 0.12$ vs. $0.75 \pm 1.30$ a.u, $P$ $<0.01$ ) and superior temporal gyrus (e.g., Ch15 $T$ task vs. W task $0.22 \pm 0.64$ vs. $0.76 \pm 0.84$ a.u, $P<0.01)$ than $T$ task. There were no significant differences in those regions between $T$ task and TW task.

\section{Conclusion}

The findings indicated that walking on a low speed requires less cognitive resources from the prefrontal cortex, while the temporal lobe is involved. When walking while texting on a smartphone, the brain areas (temporopolar area and superior temporal gyrus) involved in gait were activated, and areas in prefrontal cortex were also activated. Thus, more cognitive resources were allocated to smartphone texting during the dual task.

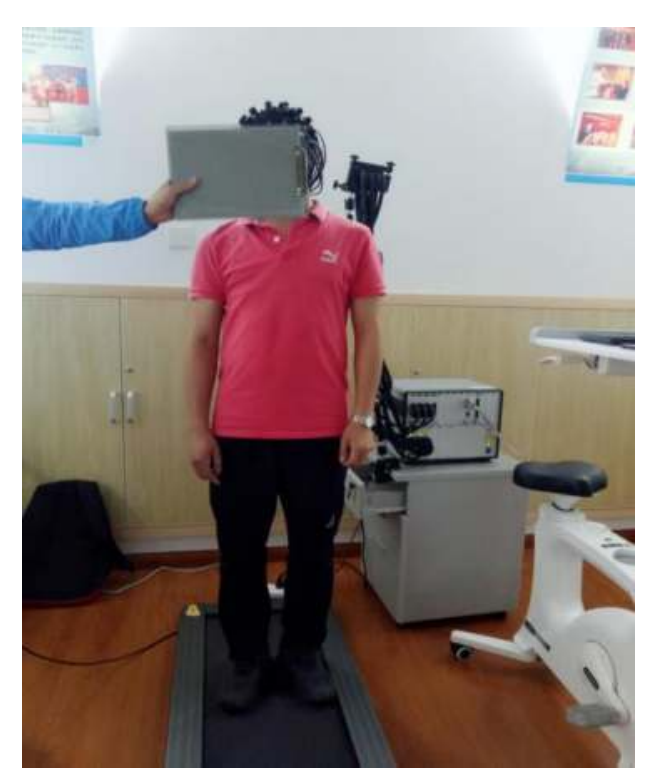

